\title{
Mapeamento e classificação do solo fazendo uso de índices de vegetação e arquitetura de aprendizagem profunda
}

\author{
Fábio Leandro Janiszevski \\ Universidade Tecnológica Federal do \\ Paraná \\ Guarapuava, Brasil \\ janiszevski@utfpr.edu.br
}

\author{
Pedro Luiz de Paula Filho \\ Universidade Tecnológica Federal do \\ Paraná \\ Medianeira, Brasil \\ pedrol@utfpr.edu.br
}

\author{
Augusto Vaghetti Luchese \\ Universidade Federal do Paraná \\ Palotina, Guarapuava \\ aluchese@ufpr.br
}

\begin{abstract}
This research aimed to develop a system with the use of multi-spectral images captured via UAV in different color spectra, and to classify them using the CNN deep learning technique, making it possible to correlate the images according to vegetation indices and soil properties. An average accuracy of $63.54 \%$ for $K$ and $75.00 \%$ for $P$, stand out the indexes CARI, MCARI_OSAVI, CHLGREEN, FE3, NORMR e MCARI to classifying K, and GEMI, MYVI, TC_YVIMSS, DATT4, FE3 e NGRDI to classifying $P$. Thus, it was observed that the methods used and data obtained in this research, make possible the proposed objective, with results that can help the identification of approximate soil properties.

Resumo- A presente pesquisa teve por objetivo desenvolver um sistema com o uso de imagens multi espectrais capturadas via VANT em diferentes espectros de cor, e classificá-las mediante técnica de aprendizagem profunda CNN, possibilitando correlacionar as imagens conforme índices de vegetação e propriedades do solo. Foi obtida uma acurácia média de $63,54 \%$ para $K$ e $75,00 \%$ para $P$, destacando-se os índices CARI, MCARI_OSAVI, CHLGREEN, FE3, NORMR e MCARI para classificar K, e GEMI, MYVI, TC_YVIMSS, DATT4, FE3 e NGRDI para classificar $P$. Desta forma, observa-se que os métodos utilizados e dados obtidos nesta pesquisa, viabilizam o objetivo proposto, com resultados que podem auxiliar a identificação de propriedades de solo aproximadas.

Palavras-chave- Análise química e física do solo; rede neural convolucional; propriedades do solo; índices de vegetação; índices espectrais.
\end{abstract}

\section{INTRODUÇÃO}

A agricultura vem passando por diversos processos de transformação decorrente das inovações tecnológicas. Contudo, ainda é um problema desafiador, surgindo a necessidade do desenvolvimento de tecnologias para um melhor aproveitamento das áreas agrícolas e um resultado otimizado da colheita [3].

Para tanto, o uso de imagens de sensoriamento remoto (SR) em regiões agrícolas, aliado aos métodos de aprendizado de máquina (AM) possibilita criar novas oportunidades para desvendar, quantificar e entender processos intensivos em dados em ambientes operacionais agrícolas, como apresentado por Pantazi et al. [12] e Su et al. [15]. Recentemente, devido a variabilidade existente em regiões agrícolas, as técnicas de AM baseadas em arquiteturas profundas apresentam viabilidade em estudos de classificação de uso do solo e estado do plantio. Essas técnicas são capazes de reconhecer padrões extremamente complexos e são adaptáveis a distorções e transformações geométricas [9]. Porém, em estudos voltados para determinação do estado do plantio agrícola, apenas informação espectral extraída por meio de uma arquitetura profunda pode não ser suficiente na tomada de decisão, necessitando análises química e física dos solos. Estas etapas tornam-se desafiadoras pois, geralmente os pesquisadores trabalham com objetivos separados, seja para classificar as propriedades químicas/físicas do solo [11], ou avaliar a cobertura e uso da área de estudo [5; 8].

Considerando ainda que, as imagens de SR podem apresentar diferentes comportamentos espectrais, a rotulação das áreas pode ser não confiável, pois depende-se da análise de um especialista, e isto é subjetivo. Desta forma, o uso de análise química e física dos solos em pontos prédeterminados na área de estudo possibilita efetuar a rotulação do estado do solo com base nos teores de argila, acidez, cálcio, dentre outras propriedades [18].

Diante deste exposto, este trabalho tem como objetivo desenvolver uma solução computacional baseada em aprendizagem profunda que, mediante imagens obtidas por VANT, permita identificar os melhores índices de reflectância para analisar as propriedades de solo Potássio (K) e Fósforo (P).

\section{TRABALHOS CORRELATOS}

Diversos artigos tem sido publicados nos últimos anos para resolução de problemas de mapeamento do solo e análise de propriedades físico-químicas [14; 7]. Alguns se dedicam a encontrar correlações com imagens de sensoriamento remoto tais como Qiang Ma et al. [13], Fasheng [4], Zanzarini et al. [18], Dong et al. [2], Sumiharto e Hardiyanto [16] e Dong et al. [1].

Qiang Ma et al. [13] analisaram o relacionamento entre dados de sensoriamento remoto e propriedades físicoquímicas do solo. Os autores realizaram pré-processamento por diferentes índices de vegetação. O relacionamento foi avaliado com correlação canônica e outros métodos de 
estatística multivariada. Nos experimentos observou-se que o SDI foi o melhor índice para avaliar o status de nutrientes do solo. Porém a correlação poderia ser avaliada utilizando resolução espectral e espacial mais alta para aprimorar a precisão do estudo. Em Fasheng et al. [4] tiveram por objetivo mapear a matéria orgânica do solo utilizando amostras de GPS e imagem Landsat. Os autores realizaram etapas de pré-processamento e extraíram características espectrais da imagem. Os métodos de regressão não linear e geoestatística para encontrar as correlações foram aplicados. Os resultados foram consistentes, porém alta resolução espectral e imagens hiperespectrais poderiam ser investigadas para o modelo proposto. Zanzarini et al. [18] analisaram uma imagem Landsat5 a fim de fazer correlação espacial do NDVI com teores de argila, fósforo e $\mathrm{pH}$. Para isso, os valores de NDVI foram cruzados com os atributos do solo por geoestatística. Os autores concluíram que o NDVI se correlaciona com argila e fósforo. Porém, para encontrar correlação com pH os autores poderiam ter analisado outros índices da literatura.

Kumar et al. [7] buscaram determinar o $\mathrm{pH}$ de amostras de solo por fotografias. As imagens foram convertidas e transformadas. Valores de RGB dos pixels foram extraídos e uma correlação entre cada amostra foi calculada. Esse formato de correlação pode ser útil na determinação do $\mathrm{pH}$ de diferentes tipos de solos. Portanto, os autores poderiam realizar o processo com diferentes propriedades.

Regalado e Cruz [14] construíram um dispositivo para determinar o teor de nutrientes do solo. Esse dispositivo foi construído com Arduino e sensores de cor e luminosidade. O teste Chi-quadrado foi utilizado para avaliação dos resultados.

$\mathrm{Xu}$ et al. [17] analisaram as correlações de índices de potássio permutável com diferentes imagens de satélite. Após realizarem calibração radiométrica e geométrica, atributos multiespectrais, topográficos e geográficos foram extraídos. Com um modelo bayesiano, foi possível fazer as predições de potássio permutável na área de estudo. Diferentes índices de reflectância foram avaliados, demonstrando correlação alta do potássio com índices Crust Index (CI), ARVI e BSI. A pesquisa demonstrou que os modelos de previsão de solo baseados em SR têm alto potencial a ser promovido para os pequenos agricultores e os ajudam a desenvolver um esquema de manejo do solo sustentável e específico do local.

Dong et al. [2] utilizaram Random Forest (RF) e redes neurais artificiais para prever o fósforo disponível no solo com base em parcelas geográficas. Eles extraíram 277 amostras de solo e, por meio de algoritmo RFC extraíram 69.562 regiões do mapa. Os resultados foram avaliados por diferentes indicadores. Contudo, como sugerido pelos autores, mais parâmetros do solo devem ser avaliados para ser um mapeamento efetivo. Já Sumiharto e Hardiyanto [16] tiveram por objetivo medir o teor de nutrientes de nitrogênio, fósforo e potássio por diferentes modelos de rede backpropagation e atributos binarizados. Foram utilizadas imagens com diferentes ângulos e distâncias, préprocessadas por diferentes métodos. Além disso, os autores avaliaram diferentes parâmetros da rede backpropagation para encontrar o melhor resultado, sendo mais rápido que testes de laboratório. Contudo, os autores poderiam incluir mais nutrientes e evitar trabalhar com dados binarizados.

Dong et al. [1] realizaram mapeamento do solo em diferentes ambientes e nutrientes típicos da região. Assim, utilizaram imagem que contém 4 bandas espectrais, diferentes atributos do terreno e 4 atributos de clima, que resultaram 130.923 unidades para mapeamento. A imagem foi utilizada como entrada para um algoritmo de aprendizagem profunda, além de outros pré-processamentos para construir as unidades de mapeamento. O algoritmo Random Forest foi utilizado como modelo e avaliado mediante diferentes indicadores estatísticos. Os experimentos mostraram que o modelo pode fornecer melhor gerenciamento das unidades avaliadas. Porém, os autores realizaram mapeamento baseado em grade, e destaca-se que a previsão de parâmetros ambientais é mais desafiadora

\section{MATERIAL E MÉTODOS}

Para esse projeto, as áreas de estudo estão localizadas no estado do Paraná, na porção Sul, situadas no município Paulo Frontin, que possui uma área de $369,210 \mathrm{~km}^{2}$, a uma latitude $26^{\circ} 02^{\prime} 24^{\prime \prime}$ sul e a uma longitude $50^{\circ} 50^{\prime} 09^{\prime \prime}$ oeste, estando a uma altitude de $778 \mathrm{~m}$. A cidade faz parte da bacia hidrográfica do rio Iguaçu. Na Figura 1 está ilustrada a localização das áreas de estudo (A e B) escolhidas para o desenvolvimento deste trabalho. A primeira área (região A) possui 18,7 hectares de plantio. A área de estudo B possui 5,7 hectares de plantio.

Os softwares utilizados foram:

- Aplicativo android "DroneDeploy" para criar os planos de vôo;

- Sistema Operacional Linux derivativo da distribuição Debian;

- Metashape, versão 1.6.3, para correções e calibração das cores das imagens e ortorretificação;

- Ambiente de desenvolvimento Python com dependências;

- Frameworks de aprendizagem profunda Caffe versão 0.16 e Digits 6.0;

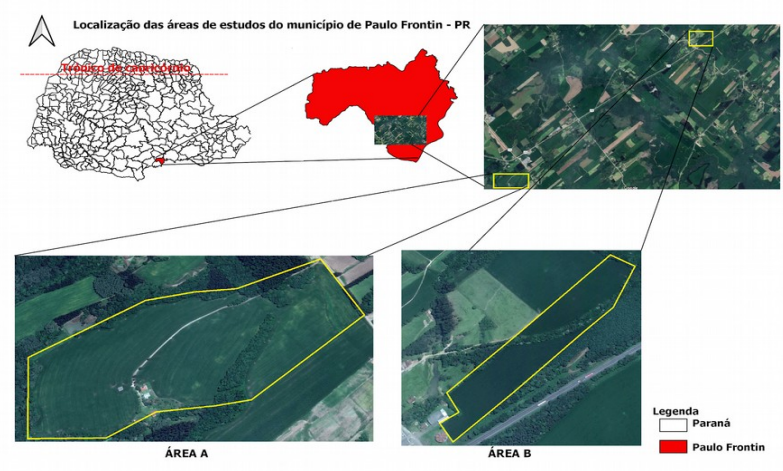

Fig. 1. Localização das áreas de estudo

Os equipamentos utilizados foram:

- $\quad$ Smartphone Android Samsung S6 Edge;

- Notebook Acer Predator Helios 300, com um processador Inter Core i7 7700HQ e 32GB de memória RAM DDR4;

- Placa de vídeo NVIDIA GTX 1060 6GB e armazenamento de 512GB em SSD e 2TB em HD;

- Veículo Aéreo Não Tripulado (VANT) modelo DJI Phantom 4 PRO para aquisição de imagens. O VANT Phantom 4 PRO é constituído por 4 motores 
e 4 escs, 1 controlador de vôo, 3 baterias, 1 módulo de sistema de navegação global por satélite (ou Global Navigation Satellite System (GNSS)), 1 controle remoto com telemetria, fuselagem, 1 suporte para a câmera Parrot Sequoia;

- 1 Câmera modelo Parrot Sequoia com 4 bandas espectrais (verde, vermelho, vermelho próximo e infravermelho próximo);

- Alvo de calibração de imagens modelo AIRINOV AIRCALIB SN: 3344;

- $\quad$ HD Externo Seagate DJI Fly Drive 2TB;

- 1 Cartão Micro SD SanDisk Extreme 32GB.

Os métodos foram divididos pelas seguintes etapas: coleta e análise química de amostras de solo, aquisição de imagens, pré-processamento de imagens, cálculo dos índices de reflectância e treinamento da CNN, sendo eles descritos na sequência.

Com o auxílio do software "Coleta de amostras" da Farmis ${ }^{1}$ foram gerados 64 pontos de amostragem a serem realizadas as coletas de solo considerando as áreas de estudo $\mathrm{A}(57)$ e $\mathrm{B}(7)$. Destas, foram selecionados 15 pontos, pela facilidade de acesso, sendo 9 pontos da área de estudo A para identificação visual aérea da amostragem, e 6 pontos da área de estudo $\mathrm{B}$, as quais foram utilizadas nos experimentos deste trabalho. A Figura 2 mostra a metodologia adotada para coleta dos pontos de amostragem.

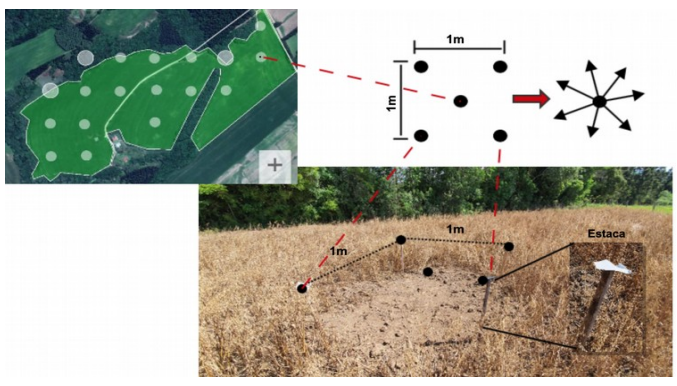

Fig. 2. Metodologia para coleta de amostras em campo

A coleta das amostras de solo foi feita com um trado calador, a uma profundidade de $10 \mathrm{~cm}$, até sete vezes em formato estrela, para se obter aproximadamente 300 gramas de solo por amostra. As seguintes propriedades químicas foram analisadas pelo laboratório com base nas amostras de solo coletadas: matéria orgânica (MO), $\mathrm{pH}$ em cloreto de cálcio $(\mathrm{Cacl} 2)$, cloreto em SMP, fósforo $(\mathrm{P})$, potássio $(\mathrm{K})$, cálcio (Ca), magnésio (Mg), alumínio (Al), saturações por bases e por alumínio. Com os resultados destas análises, pode-se selecionar as propriedades e a base de dados pode ser rotulada. Para os experimentos deste trabalho, somente as informações de fósforo (P) e potássio (K) foram utilizadas.

Os planos de vôo executados foram configurados com uma altura de vôo em 90 metros a partir do ponto de decolagem, sobreposição frontal de $85 \%$ e lateral de $70 \%$ em cada captura de imagem, e uma velocidade de cruzeiro máxima de $11 \mathrm{~m} / \mathrm{s}$, as quais foram configuradas com o auxílio do aplicativo DroneDeploy e ajustadas conforme o tempo de vôo para se adequar ao VANT utilizado.

A aquisição de imagens na área de estudo A, particionada em 3 segmentos focados nos 9 pontos visualmente identificáveis, totalizou 366 imagens da câmera Parrot Sequoia. Na região $\mathrm{B}$, capturou-se 1710 imagens, cobrindo toda a área da região. Para tanto, realizou-se etapas de calibração.
Na etapa de processamento de imagens, foi realizada a ortorretificação a fim de eliminar distorções causadas especialmente pela posição da câmera e pela superfície física da Terra (relevo e curvatura) [10]. Na sequência, foram avaliados os índices de reflectância que foram possíveis de serem aplicados nas áreas de estudo, conforme a formulação das bandas espectrais da câmera Parrot Sequoia. Assim, foram considerados 70 índices de reflectâncias, como por exemplo NDVI, GNDVI, MCARI, LCI, NDRE, dentre outros.

Essa pesquisa foi realizada na plataforma Caffe e contêm funções para criação de dados, treinamento de arquiteturas e análise de resultados.

A primeira etapa foi preparar as imagens capturadas das áreas de estudo, conforme a seleção das 15 amostras de solo coletadas e rotuladas, destas, 11 foram para treino, com 7 amostras da área de estudo A e 4 amostras da área de estudo B. Para teste, foram selecionadas 4 amostras, sendo 2 amostras da área A e 2 amostras da área B. As imagens para o treinamento da CNN foram geradas baseando-se na sobreposição dos mapas ortorretificados com combinações de índices de reflectância e rotulação.

Assim, imagens de tamanho 256x256 foram criadas para serem utilizadas como dados de entrada da CNN. Para o treinamento foi utilizada a arquitetura de rede Alexnet proposta por Krizhevsky et al. [6]. Conforme mostra a Figura 3, essa rede é composta por 8 camadas, sendo 5 convolucionais e 3 totalmente conectadas para formar o mapa de características. Nessa arquitetura, das imagens, são extraídos patches randômicos de 224x224 a partir de imagens 256x256 com 3 dimensões. Para os experimentos, os valores de entrada e saída foram modificados na arquitetura.

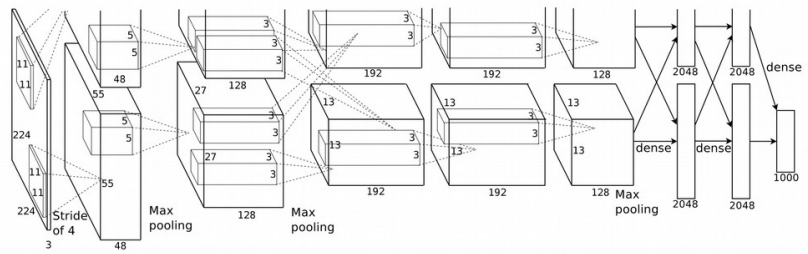

Fig. 3. Representação da arquitetura Alexnet de Krizhevsky et al. [6]

Para a entrada foram consideradas a permutação três a três dos 70 índices de reflectância. Já a saída tem como resultado a taxa de classificação em cada classe conforme a propriedade química treinada. Os hiperparâmetros utilizados para treinamento foram, taxa de aprendizagem 0,01 , o momentum 0,9, 5 épocas e a função de otimização Stochastic Gradient Descent (SGD). A análise dos resultados foi realizada mediante matriz de confusão para verificar o desempenho do classificador.

\section{RESUltAdOS E DISCUSSÃO}

Para o entendimento da distribuição dos resultados das análises químicas das amostras coletadas, foram calculados a média, variância, desvio padrão, mínimo e máximo para cada propriedade química. Os resultados destes cálculos estão apresentados na Tabela I. Com estes resultados, foi feita a rotulagem de cada amostra conforme a sua propriedade.

TABELA I

ESTATÍSTICAS DESCRITIVAS DAS AMOSTRAS DE SOLO

\begin{tabular}{|c|c|c|}
\hline Estatística & Propriedade & \\
\hline & $\mathbf{K}$ & $\mathbf{P}$ \\
\hline & $\mathrm{cmol} / \mathrm{dm} 3$ & $\mathrm{cmol} / \mathrm{dm} 3$ \\
\hline
\end{tabular}




\begin{tabular}{|c|c|c|}
\hline Média & 0,572 & 12,137 \\
\hline Variância & 0,014 & 47,539 \\
\hline Desv. Pad. & 0,120 & 6,895 \\
\hline Mínimo & 0,280 & 3,620 \\
\hline Máximo & 0,760 & 30,600 \\
\hline
\end{tabular}

Desv. Pad: Desvio Padrão

Conforme discutido na seção de materiais e métodos, foram selecionadas 15 amostras para realização dos experimentos. As amostras de treinamento e validação da CNN foram nomeadas de: 408, 415, 434, 435, 450, 463, 464, 501, 502, 504 e 507, e para teste: 404, 433, 503 e 505. Essa separação de amostras entre treino/validação e testes foi devida a distribuição e balanceamento dos dados conforme as análises descritivas apresentadas na Tabela I.

Para aplicação dos índices, foi necessário gerar as imagens a partir das amostras selecionadas. Cada amostra gerou uma região de interesse delimitada por quatro pontos de coleta marcados pelo GPS. Essa região é utilizada para o cálculo dos índices de reflectância.

Em cada uma das amostras, foram aplicados os 70 índices de reflectância selecionados. Para exemplificar a composição final de cada imagem, a Figura 4 apresenta um mapa colorimétrico da amostra 450 da área de estudo A, com a exemplificação da aplicação dos índices MCARI2 e NDVI, que estão demonstrados nas Figuras $4 \mathrm{a}$ e 4b, respectivamente. Eles apresentam os valores calculados por cada índice espectral em seu respectivo pixel.
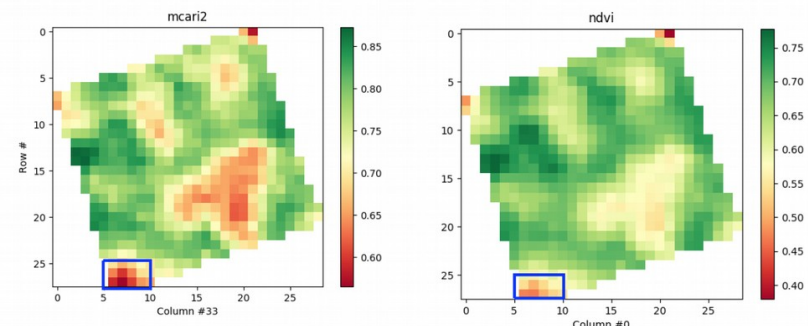

Fig. 4. Exemplo da aplicação dos índices de reflectância na amostra 450 da área de estudo A

Com a determinação das amostras para treinamento e teste, foi estabelecida uma classificação baseada no desvio padrão de cada propriedade química do solo (K e P). A partir da estatística descritiva das amostras selecionadas (Tabela I) foi possível a definição das classes. Para a definição da primeira classe foi atribuído ao limite inferior o valor mínimo encontrado, e para a definição do limite superior da classe, foi usado o limite inferior mais o valor de desvio padrão, e assim sucessivamente, classificando-os em até 4 classes, denominadas Desvio 1, Desvio 2, Desvio 3 e Desvio 4 (Dv1, Dv2, Dv3 e Dv4).

Os treinos e testes na arquitetura Alexnet foram realizados a partir das imagens geradas pelas permutações (3 a 3) dos 70 índices de reflectância selecionados, gerando 328.440 combinações possíveis por amostra, e 4.692 combinações por índice em cada posição em cada amostra, totalizando 14.076 vezes que um mesmo índice aparecerá em cada amostra. Com isso, obteve-se um total de 3.612.840 imagens, separadas em $75 \%$ para treino e $25 \%$ para validação, sendo 2.709 .630 e 903.210 de imagens respectivamente. Assim a base de dados gerada totalizou aproximadamente 151 Gigabytes para cada propriedade química.

A Tabela II apresenta a acurácia obtida pelas propriedades K e P. A média geral foi de 18,39\% de acurácia considerando todas as classes. Contudo, não houveram amostras para treino na classe Dv1 na propriedade K, e nem amostras para as classes Dv3 e Dv4 na propriedade P.

TABELA II

ACURÁCIA GERAL PARA AS PROPRIEDADES K E P CONFORME A CLASSE

\begin{tabular}{|c|c|c|}
\hline & $\mathrm{K}$ & $\mathrm{P}$ \\
\hline Dv1 & - & $24,83 \%$ \\
\hline Dv2 & $0,31 \%$ & $0 \%$ \\
\hline Dv3 & $18,86 \%$ & - \\
\hline Dv4 & $34,58 \%$ & - \\
\hline Média & $18,16 \%$ & $18,62 \%$ \\
\hline
\end{tabular}

A permutação aplicada combina um mesmo índice em posições diferentes diversas vezes, para gerar uma imagem composta de três canais (RGB). Após o treinamento, cada modelo treinado pode ser utilizado para efetuar os testes. Ou seja, as amostras puderam ser classificadas conforme cada propriedade química analisada.

Nos experimentos realizados com a propriedade potássio (K), no primeiro espectro R (Red), ao ordenar os índices pela quantidade de acertos, tem-se como relevante para a classificação os índices CARI, com 5.712 elementos classificados corretamente, de um total de 14.076 elementos possíveis. Seguindo essa mesma linha, para Red, tem-se o MCARI_OSAVI (5.372) e CHLGREEN (5.100). Já para o segundo espectro, que contempla o G (Green), observou-se que os índices FE3 (13.330), NORMR (13.060) e MCARI (9.384) se destacaram como mais relevantes. Porém, para o terceiro espectro, o B (Blue), verifica-se similaridade nos valores total da contagem entre os índices, visto que os três primeiros resultaram em DIFF1 (3.466), MTVI2 (3.466) e GOSAVI (3.466) como relevantes. Portanto, para a propriedade K os índices escolhidos para os espectros R e G são os mais relevantes para a classificação dessa propriedade. A Tabela III apresenta a matriz de confusão dos elementos selecionados para $\mathrm{R}$ e $\mathrm{G}$ e verifica-se a acurácia de $63.54 \%$ e 2.992 elementos classificados.

TABELA III

RESULTADOS FILTRADOS DA CLASSIFICAÇÃO PARA POTÁSSIO CONSIDERANDO OS ESPECTROS R E G

\begin{tabular}{|c|c|c|c|c|c|c|c|c|}
\hline & Dv1 & Dv2 & Dv3 & Dv4 & Total & Err. Inc. & Err. Oms. & Acc. \\
\hline Dv1 & 0 & 0 & 0 & 0 & 0 & - & - & - \\
\hline Dv2 & 0 & 0 & 612 & 136 & 748 & $0,00 \%$ & $100 \%$ & $0,00 \%$ \\
\hline Dv3 & 0 & 0 & 1156 & 340 & 1496 & $40,90 \%$ & $22,72 \%$ & $77,27 \%$ \\
\hline Dv4 & 0 & 0 & 0 & 748 & 748 & $63,63 \%$ & $0,00 \%$ & $100 \%$ \\
\hline Total & 0 & 0 & 1768 & 1224 & 2992 & $36,36 \%$ & $36,36 \%$ & $63,54 \%$ \\
\hline
\end{tabular}

*Err. Inc: erro de inclusão. Err. Oms.: erro de omissão. Acc: acurácia

Nos experimentos realizados para a propriedade $\mathrm{P}$, considerando apenas os três primeiros índices de cada espectro, sendo os índices mais significativos, para o primeiro espectro R (Red), GEMI (6.460), MYVI (6.460) e TC_YVIMSS (6.324) são os índices mais relevantes que os demais.

Para o segundo espectro, no G (Green), verificou-se que os índices DATT4 (14.080), FE3 (13.870) e NGRDI (13.260) são os que possuem maior relevância para o P. E por fim, foi observada uma contagem semelhante entre os índices no terceiro espectro (B - Blue), sendo os três primeiros TCARI (3.556), TCARI2 (3.553) e SBL (3.552) os mais relevantes . Porém observa-se que não existe diferença significativa entre os índices para esse espectro. Observa-se no- 
vamente que os índices selecionados para Red e Green são relevantes para a propriedade P. A Tabela IV apresenta a matriz de confusão dos elementos selecionados para $R$ e $G$ na propriedade $\mathrm{P}$.

Assim, ao selecionar apenas os melhores índices para $\mathrm{R}$ e G, tem-se 2.448 elementos classificados, e destes, consegue-se uma acurácia de 75,00\%.

TABELA IV

RESULTADOS FILTRADOS DA CLASSIFICAÇÃO PARA FÓSFORO CONSIDERANDO OS ESPECTROS R E G

\begin{tabular}{|c|c|c|c|c|c|c|c|c|}
\hline & Dv1 & Dv2 & Dv3 & Dv4 & Total & Err. Inc. & Err. Oms. & Acc. \\
\hline Dv1 & 1836 & 0 & 0 & 0 & 1836 & $11,11 \%$ & $0 \%$ & $100 \%$ \\
\hline Dv2 & 204 & 0 & 204 & 204 & 612 & $0,00 \%$ & $100 \%$ & $0,00 \%$ \\
\hline Dv3 & 0 & 0 & 0 & 0 & 0 & - & - & - \\
\hline Dv4 & 0 & 0 & 0 & 0 & 0 & - & - & - \\
\hline Total & 2040 & 0 & 204 & 204 & 2448 & $25,00 \%$ & $25,00 \%$ & $75,00 \%$ \\
\hline
\end{tabular}

*Err. Inc: erro de inclusão. Err. Oms.: erro de omissão. Acc: acurácia

\section{CONCLUSÃO}

A presente pesquisa teve por objetivo desenvolver uma solução computacional baseada em aprendizagem profunda que, com o uso de imagens capturadas via VANT, possibilitasse avaliar os melhores índices de reflectância para as propriedades $\mathrm{K}$ e $\mathrm{P}$.

A abordagem de aprendizagem profunda possibilitou a extração de diferentes mapas de características, reduzindo a complexidade de análise de métodos manuais para extração de características. Isso tornou possível o treinamento dessas imagens geradas, que são normalmente complexas para o processamento de imagens de SR. Assim, foram obtidas 15 amostras do solo visualmente identificáveis nas imagens aéreas, o que é pouco para uma rede neural profunda.

Apesar disso, os resultados foram bastante promissores. Conjuntos de 328.440 imagens foram gerados a partir de cada amostra de solo/imagem, conforme alternância de índices de reflectância conforme o espectro da imagem RGB. Com isso, foi possível detectar impactos de diferentes índices de reflectância em duas propriedades químicas, verificando quais foram relevantes para cada propriedade.

Contudo, outros experimentos podem ser incluídos a essa base de dados. Uma alternativa seria o uso de métodos de interpolação de dados, como a krigagem ou o semivariograma, o que permitiria obter valores aproximados das propriedades químicas do solo, em pontos onde não é possível identificar visualmente as amostras de solo, ou onde não foram realizadas coletas físicas, aumentando assim o número de amostras. Além disso, como trabalhos futuros pretende-se classificar todas as propriedades obtidas por amostra de solo, realizar data augmentation para aumentar a base de dados, realizar a correlação entre os índices para encontrar aqueles que possuem maior relevância para todas as propriedades químicas.

\section{REFERENCIAS}

[1] DONG, W.; SUN, Y.; LUO, J. Fine mapping of key soil nutrient content using highresolution remote sensing image to support precision agriculture in Northwest China. In:2019 8th International Conference on Agro-Geoinformatics (Agro-Geoinformatics).Istanbul, Turkey:IEEE, 2019. p. 1-5.

[2] DONG, W. et al. Digital Mapping of Soil Available Phosphorus Supported by AI Technologyfor Precision Agriculture. In:2018 7th
International Conference on Agro-geoinformatics (Agrogeoinformatics). Hangzhou: IEEE, 2018.

[3] FAO; IFAD; WFP. The State of Food Insecurity in the World 2013: The Multiple Dimensions of Food Security. [S.l.]: FAO, 2014. ISBN 9251079161.

[4] FASHENG Zhang et al. Mapping surface soil organic matter based on multispectralimage. In:2010 International Conference on Image Analysis and Signal Processing.Zhejiang, China:IEEE, 2010.

[5] FERENTINOS, K. P. Deep learning models for plant disease detection and diagnosis. Computers and Electronics in Agriculture, v. 145, p. 311 - 318, 2018. ISSN 0168-1699.

[6] KRIZHEVSKY, A.; SUTSKEVER, I.; HINTON, G. E. Imagenet classification with deep convolutional neural networks. In: Proceedings of the 25th International Conference on Neural Information Processing Systems - Volume 1. USA: Curran Associates Inc., 2012.

[7] KUMAR, V. et al. Determination of soil $\mathrm{pH}$ by using digital image processing technique.JANS, v. 6, n. 1, p. 14-18, jun. 2014.

[8] KUSSUL, N. et al. Deep learning classification of land cover and crop types using remote sensing data. IEEE Geoscience and Remote Sensing Letters, PP, p. 1-5, 032017.

[9] LECUN, Y.; KAVAKCUOGLU, K.; FARABET, C. Convolutional networks and applications in vision. In: Proceedings of ISCAS. Paris: [s.n.], 2010. p. 253-256.

[10] MITISHITA, P. P. e Alzir Antunes e E. Ortoretificação de imagens de alta resolução utilizando os modelos apm (affine projection model) e rpc (rational polynomial coeffycient). Boletim de Ciências Geodésicas, v. 13, n. 1, 2007. ISSN 1982-2170.

[11] PADARIAN, J.; MINASNY, B.; MCBRATNEY, A. Using deep learning to predict soil properties from regional spectral data. p. e00198, 112018

[12] PANTAZI, X. et al. Wheat yield prediction using machine learning and advanced sensing techniques. Computers and Electronics in Agriculture, v. 121, p. 57 - 65, 2016. ISSN 0168-1699.

[13] QIANG Ma; WANTAI Yu; HUA Zhou. The relationship between soil nutrient properties andremote sensing indices in the Phaeozem region of Northeast China. In:2010 SecondInternational Conference on Computational Intelligence and Natural Computing.Wuhan, China:IEEE, 2010. p. 109-112.

[14] REGALADO, R. G.; CRUZ, J. C. D. Soil pH and nutrient (Nitrogen, Phosphorusand Potassium) analyzer using colorimetry. In:2016 IEEE Region 10 Conference(TENCON). Singapore: IEEE, 2016.

[15] SU, Y. xue; XU, H.; YAN, L. jiao. Support vector machine-based open crop model (sbocm): Case of rice production in china. Saudi Journal of Biological Sciences, v. 24, n. 3, p. 537 - 547, 2017. ISSN 1319-562X.

[16] SUMIHARTO, R.; HARDIYANTO, R. NPK Soil Nutrient Measurement Prototype Based onLocal Binary Pattern And BackPropagation. In:2018 IEEE International Conference onInternet of Things and Intelligence System (IOTAIS). Bali: IEEE, 2018.

[17] XU, Q. et al. Extraction of leaf biophysical attributes based on a computer graphic-basedalgorithm using terrestrial laser scanning data. Remote Sensing, v. 11, p. 15, 122018.

[18] ZANZARINI, F. V. et al. Correlação espacial do Índice de vegetação (NDVI) de imagem Landsat/ETM+ com atributos do solo. Revista Brasileira de Engenharia Agrícola e Ambiental, scielo, v. 17, p. 608 614, 06 2013. ISSN 1415-4366. 